\title{
Applying Multifractal Spectrum Theory to Fingerprint Features Recognition
}

\author{
Hai Ming Ni, Da Wei Qi* and Hongbo Mu \\ College of Science, Northeast Forestry University, Hexing Road 26, Harbin, \\ Heilongjiang Province, 150040, PR China \\ * Correspending Author, Tel.: 045182191571, fax: 045182191571. E-mail: \\ qidw9806@126.com
}

\begin{abstract}
Fingerprint features recognition which can be used to distinguish between individuals is an intriguing study with many potential applications. In this paper, a new method for fingerprint recognition based on multifractal spectrum theory was proposed. The recognition process can be divided into the following main steps: (1) Extracting the core point in fingerprint; (2) Fragmenting the fingerprint image to get a subimage with fixed size; (3) Thinning the fingerprint image by using an improved OPTA algorithm; (4) Segmenting the curves in fingerprint image into digital straight segments with normalized straight length threshold; (5) Selecting the appropriate dividing scale to segment the processed fingerprint image; (6) Calculating and analyzing the multifractal spectrum curve $\alpha-f(\alpha)$; (7) Fitting curve equation and extracting the characteristic parameters of $\alpha-f(\alpha)$; (8) Finally, the parameters matching and fingerprint feature recognition. A large number of experimental results show that our method is effective.
\end{abstract}

Keywords: Multifractal spectrum; Fingerprint capture; Digital straight segment; Fingerprint recognition

\section{Introduction}

Among all the global features, orientation field is one of the most widely used. Compared with the minutiae characteristics, the orientation field is more stable in the process of fingerprint feature extraction and feature matching. In recent years, the orientation field features of fingerprints had been studied by many researchers [1-2]. The orientation field of fingerprints is usually defined as the local orientation of ridge and valley. There is an orthogonal relationship between the orientation field and the gradient field. Therefore, the orientation field is usually computed by calculating the gradient field. However, the orientation field is not able to automatically capture the specially structural information of local regions. Hence, calculating the orientation field directly is not convenient for fingerprint matching. To overcome these problems in orientation field methods, a method to exact features with stable orientations based on long digital straight segments was proposed by Jiang et al [3]. Through this method, the extracted curves from a thinned fingerprint image are decomposed into digital straight segments. Although, this method have many advantages (e.g., more stable, less storage, more accurate marching) compared with the traditional orientation field methods, the marching of digital straight segments are also very difficult.

Multifractal spectrum features have been widely used in signal analysis, financial analysis, object modeling and image classification, etc., [4-5]. In this paper, we proposed a new fingerprint recognition method by analyzing the orientation field of decomposed digital 
straight segments based on multifractal spectrum theory. The multifractal spectrum of processed fingerprint was calculated by using inclination functions. An quadratic equation was extracted from the multifractal spectrum curve $\alpha-f(\alpha)$, which can represent the features of fingerprint. A large number of experimental results show that this method is feasible.

\section{Image Capture and Feature Extraction for Fingerprint}

\subsection{Core Point Detection}

The core point is defined as a concentrate region where the ridges' curvature is converging to a local maximum [6]. The core point has been widely used as reference point for fingerprint alignment [7]. Figure 1 is a fingerprint image to be processed. First of all, we implement fingerprint image enhancement processing and the result shows in Figure 2.

Then, a composite Gauss wave filter [8] is used in this paper. It is defined as:

$$
\begin{aligned}
& h_{1}=(x+i y)^{m} g(x, y), \\
& g(x, y)=\exp \left(-\left(x^{2}+y^{2}\right) / 2 \sigma^{2}\right)
\end{aligned}
$$

This operation is not directly applied to the fingerprint image, but used in the composite orientation field of fingerprint image. The composite orientation field is defined as:

$$
z(x, y)=\left(f_{x}+i f_{y}\right)^{2}
$$

where $f_{x}=\partial z(x, y) / \partial x, f_{y}=\partial z(x, y) / \partial y$.

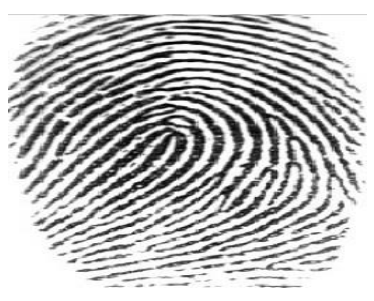

Figure 1. The Original Fingerprint Image

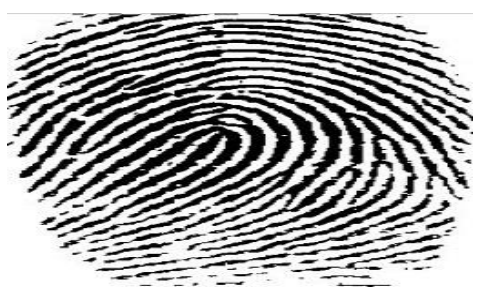

Figure 2. The Fingerprint Image after Enhancement Processing

Finally, we find the core point through calculating the orientation field and smooth operation by using the following formulas.

$$
\begin{aligned}
& \phi_{x}(i, j)=\cos [2 \times o(i, j)] \\
& \phi_{y}(i, j)=\sin [2 \times o(i, j)]
\end{aligned}
$$

where $o(i, j)$ is the calculated orientation.

$$
\begin{aligned}
& \phi_{x}^{\prime}(i, j)=\sum_{u=-w_{\phi} / 2 v=-w_{\phi} / 2}^{w_{\phi} / 2} \sum_{w_{\phi} / 2}^{w_{\phi} / 2} W(u, v) \cdot \phi_{x}(i-u w, j-v w) \\
& \phi_{y}^{\prime}(i, j)=\sum_{u=-w_{\phi} / 2 v}^{w_{\phi} / 2} \sum_{v=-w_{\phi} / 2}^{w^{\prime}} W(u, v) \cdot \phi_{y}(i-u w, j-v w)
\end{aligned}
$$


where $W(u, v)$ is the orientation field after two dimensional low pass filter smooth operation.

$$
o^{\prime}(i, j)=1 / 2 \times \tan ^{-1}\left(\phi_{y}^{\prime}(i, j) / \phi_{x}^{\prime}(i, j)\right)
$$

The core point of the original fingerprint image which is extracted by using this method is shown in Figure 3.

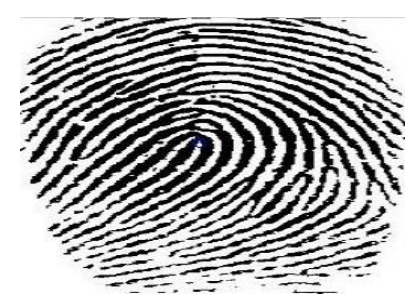

Figure 3. Fingerprint Image after Core Point Extracting

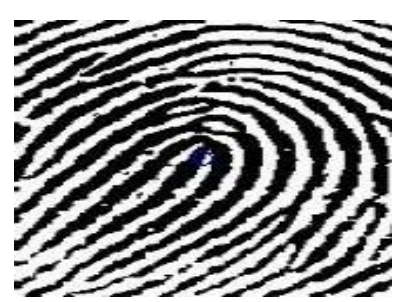

Figure 4. Cropped Image around Core Point

\subsection{Image Segmentation and Fingerprint Thinning}

In order to improve the accuracy of fingerprint matching, we set a certain size threshold of fingerprint by cutting the fingerprint image. The image of size 161 by 161 pixels around the core point has been cropped as shown in Figure 4. With the development of fingerprint recognition technology, researchers have proposed many effective fingerprint thinning methods [8-9]. In this paper, the improved one pass thinning algorithm proposed in [10] is used. In this algorithm, the efficiency of the fingerprint thinning is improved by using the new delete and reserve templates. Figure 5-6 are the schematic diagrams of the new delete and reserve templates, respectively. Figure 7 is the fingerprint image after thinning.

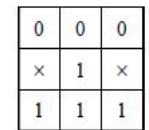

(a)

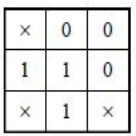

(e)

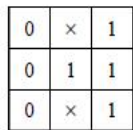

(b)

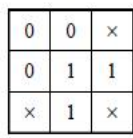

(f)

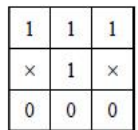

(c)

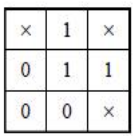

(g)
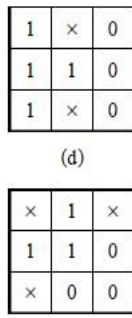

(h)

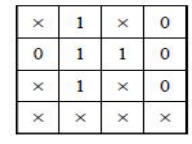

(a)

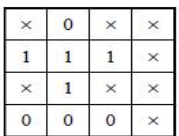

(d)

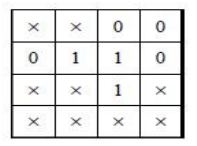

(b)

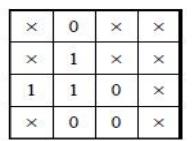

(e)
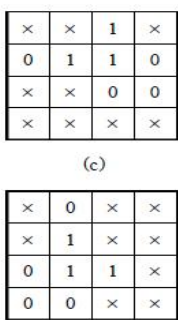

$(f)$
Figure 5. The Delete Templates of the New One Pass Thinning Algorithm
Figure 6. The Reserve Templates of the New One Pass Thinning Algorithm

\subsection{Digital Straight Segments}

Digital straight lines and digital straight segments are widely studied in the past decades. Several effective algorithms have been proposed for segment a curve into digital straight segments [11-13]. In this paper, the digital straight segments algorithm proposed in [3] is used. In this literature, the fingerprint image is divided into long digital straight lines according to a certain pixel threshold $\mathrm{T}$. All curves can be segmented by these parameters $\{L(s), \theta(s)$, Length $(s) \mid \mathrm{s}=1,2, \ldots, \mathrm{N}\}$. Where $\mathrm{N}$ is the total number of segments, 
$L(s)=\left(q_{1} q_{2} \cdots q_{M}\right),\left\{q_{i}=\left(x_{i}, y_{i}\right) \mid \mathrm{i}=1,2, \ldots, \mathbf{M}\right\}, q_{i}$ is the pixel value of ith point, $\theta(s)$ is the orientation angle of the digital straight segment $L(s), \theta(s) \in[0, \pi)$, Length $(s)$ is the length of the digital straight segment $L(s)$. If the length of one segment is longer than the threshold T, namely Length $(s) \geq T$, the orientation angle of this segment is regarded as stable. In our experiments, the threshold $\mathrm{T}$ is set to 15 pixels. In fingerprint image, the segments longer than 15pixels are shown in Figure 8.

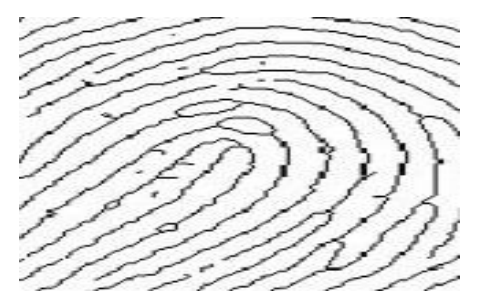

Figure 7. Fingerprint Image after Thinning

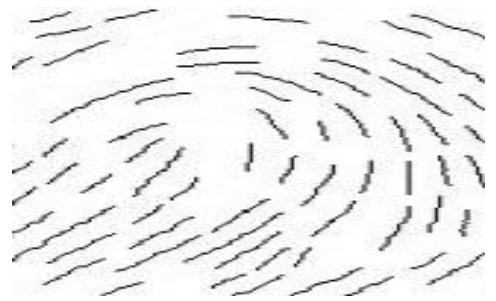

Figure 8. Fingerprint Image after Straight Cutting

\section{Multifractal Spectrum Fingerprint Recognition Method}

Multifractal (also called multiple scale or complex fractal) is often used to generalize a fractal system which cannot be totally described by using a single exponent (the fractal dimension); it is from the local property to study the characteristics of the shape. In this section, we analyze the multifractal spectrum features of fingerprint images and propose a new fingerprint recognition method based on multifractal spectrum theory.

First of all, we use the box-counting method to deal with the fingerprint image after straight cutting in Figure. 8. A dividing scale $\delta$ is selected. The size of Figure 8 is 161 by 161 pixels. Hence, the minimum dividing scale is $1 / 161$ and the maximum dividing scale is 1 . If the dividing scale is $1 / 161$, there will only get some isolated pixels but not small curve segments. The suitable dividing scale is $1 / 161<\delta \leq 1$. If we select a certain dividing scale $\delta$, then the image Figure 8 will be divided into $(1 / \delta)^{2}$ square lattices. Next, we calculate the angle of small straight segments in each square lattice. For each square lattice $\mathrm{i}(\mathrm{i}=1,2, \ldots$, $\left.(1 / \delta)^{2}\right)$, we define the sum of inclination angles of all small straight segments as $\theta_{i}(\delta)$. Then, we can get the sum of inclination angles of all small straight segments in the fingerprint image, $\theta(\delta)=\sum_{i} \theta_{i}(\delta)$. So we get the probability function:

$$
p_{i}(\delta)=\theta_{i}(\delta) / \theta(\delta)
$$

$p_{i}(\delta)$ and $\delta$ obey the power law (the power is the fractal dimension) which depends on the dividing scale $\delta$.

$$
p_{i}(\delta) \propto \delta^{\alpha}
$$

Then, we can get the Hölder exponent $\alpha$ and the multifractal spectrum:

$$
\alpha=\sum p_{i}^{q}(\delta) \ln p_{i}(\delta) / \ln (\delta) \sum p_{i}^{q}(\delta)
$$




$$
f(\alpha)=\left\{\sum\left[p_{i}^{q}(\delta) \ln p_{i}(\delta)\right] / \ln (\delta) \sum p_{i}^{q}(\delta)\right\} \times q-\ln \left[\sum p_{i}^{q}(\delta) / \ln (\delta)\right.
$$

where $q$ is the weighting factor.

The multifractal spectrum curve $f(\alpha)$ is a unimodal function. This function can be expressed as a quadratic function by using the least square fit method. The basic form of the function is as following:

$$
f(\alpha)^{\prime}=a\left(\alpha-\alpha_{0}\right)^{2}+b\left(\alpha-\alpha_{0}\right)+c
$$

Where $\alpha_{0}$ is the value of the Hölder exponent $\alpha$ when the multifractl spectrum function $f(\alpha)$ reaches the global maximum. Namely, $f_{\max }=f\left(\alpha_{0}\right)$. The parameter $\mathrm{b}$ shows the asymmetry of the multifractal spectrum curve. The parameter $W=\alpha_{\max }-\alpha_{\min }$ is bandwidth of the multifractal spectrum, where $f\left(\alpha_{\max }\right)=f\left(\alpha_{\min }\right)=0$.

Formula (12) can also be rewritten as the following form:

$$
f(\alpha)^{\prime}=a\left(\alpha-\alpha_{0}^{\prime}\right)^{2}+\mathrm{d}
$$

Where $\alpha_{0}^{\prime}$ is the real value of $\alpha_{0}$ when the function $f(\alpha)^{\prime}$ reaches the global maximum. Namely, $f_{\max }^{\prime}=f\left(\alpha_{0}^{\prime}\right) \cdot \mathrm{H}=\alpha_{0}^{\prime}-\alpha_{0}$ shows the top displacement of the multifractal spectrum curve. These four parameters $\left(f_{\max }, \mathrm{b}, \mathrm{W}, \mathrm{H}\right)$ provide a fast and accurate fingerprint recognition algorithm which based on multifractal spectrum theory.

\section{Experiments}

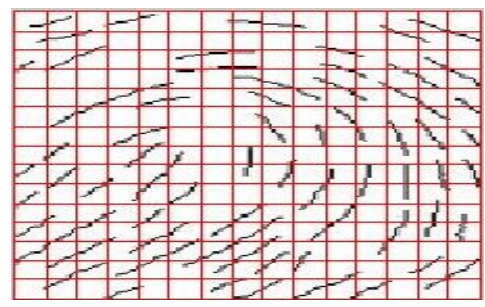

Figure 9. The Fingerprint Image after Meshing

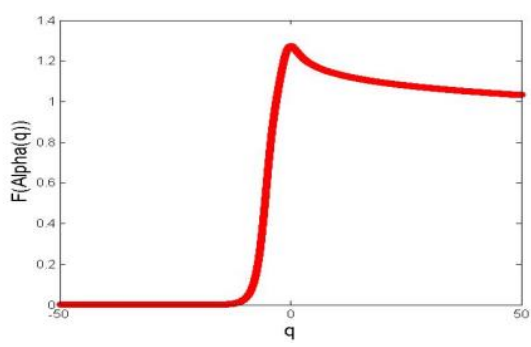

Figure 11. The Graph of the Multifractal Spectrum $f(\alpha)$ with the Value of $\boldsymbol{q}$

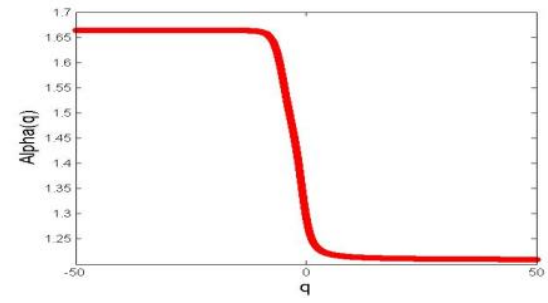

Figure 10. The Graph of the Hölder Exponent $\alpha$

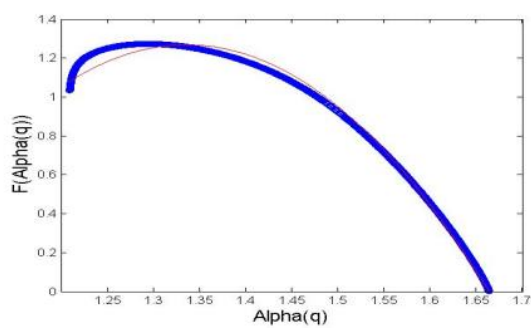

Figure 12. The Graph of the Multifractal Spectrum $f(\alpha)$ with the Value of the Hölder Exponent $\alpha$ 
In order to design an accurate multifractal fingerprint recognition system, the dividing scale $\delta$ must be determined at first. A large number of experiments show that the best dividing scale is $\delta=1 / 16$. Figure 9 is the divided fingerprint image of $\delta=1 / 16$ by using a meshing algorithm. From Figure 9 we can see when the dividing scale $\delta=1 / 16$, the straight segments of each square lattice are only three possibilities. Zero, one and two small straight line.

Once the dividing scale is determined, we can calculate the slope angle of each small straight segment in each small square lattice easily by using a MATLAB angle calculation program. And then the probability function $p_{i}(\delta)$, the Hölder exponent $\alpha$, and the multifractal spectrum $f(\alpha)$ are calculated by the formulas (8), (10) and (11). All of these parameters are obtained by using a series of '. $m$ ' files which we compile in MATLAB. Fig. 10-12 shows the graphs of these parameters.

Figure 10 is the graph of the Hölder exponent $\alpha$ with the value of q. From this graph we can get the bandwidth parameter $W=\alpha_{\max }-\alpha_{\min }=0.4533$. In Figure $11-12$, when the weighting factor $q=0$, the maximum value $f_{\max }=1.2931$ and the Hölder exponent $\alpha_{0}=1.2805$ are obtained. Figure 12 is the graph of the multifractal spectrum $f(\alpha)$ with the value of the Hölder exponent $\alpha$. There are two curves in this graph, where the blue one is the real multifractal spectrum curve of the fingerprint straight segments, and the red parabola is a quadratic fitting curve to $f(\alpha)$ around $\alpha_{0}$ by using a least square method. The quadratic function is as following:

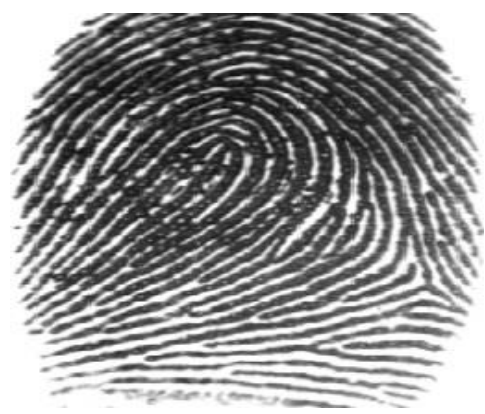

(a)

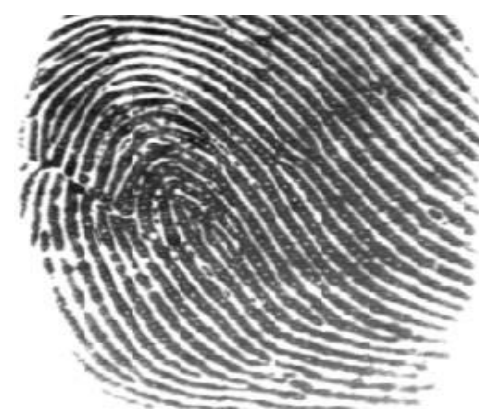

(c)

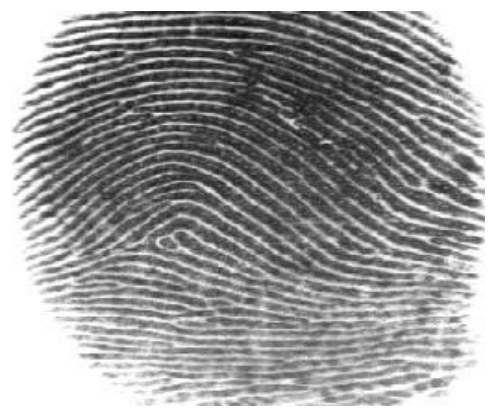

(b)

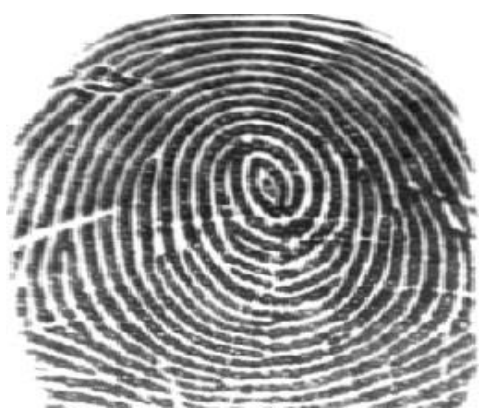

(d)

Figure 13. Four Different Fingerprints Images

$$
f(\alpha)^{\prime}=-11.7486(\alpha-1.2805)^{2}+1.2947(\alpha-1.2805)+1.2329
$$




$$
f(\alpha)^{\prime}=-11.7486(\alpha-1.3356)^{2}+1.2686
$$

Then, the asymmetry parameter $b=1.2947$ and the top displacement $\mathrm{H}=0.0551$ of the fingerprint straight segments multifractal spectrum curve are obtained.

Fingerprints are one of the most reliable and stable biological characteristic and everyone even twins have different fingerprints. Therefore, we believe that the multifractal spectrum parameters of each fingerprint should also be different from each other. Then, the four multifractal spectrum parameters of fingerprint straight segments can be used as the reference to distinguish the different fingerprints.

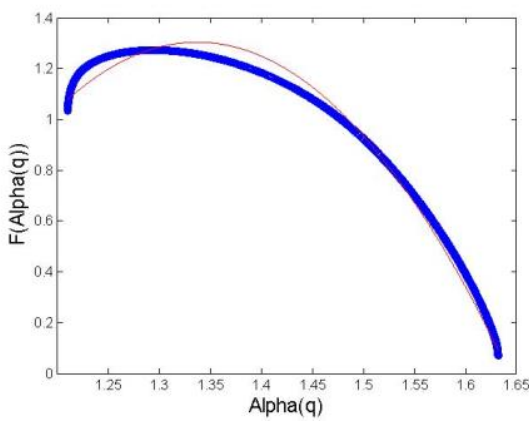

(a)

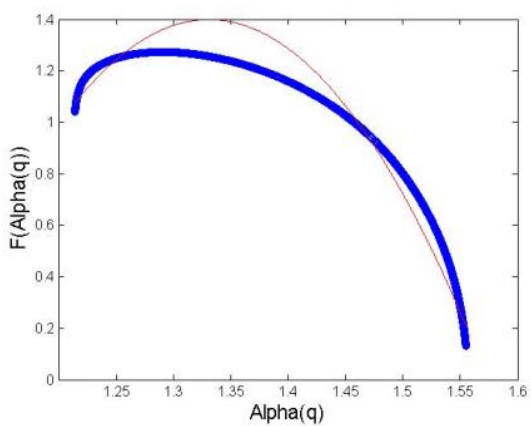

(c)

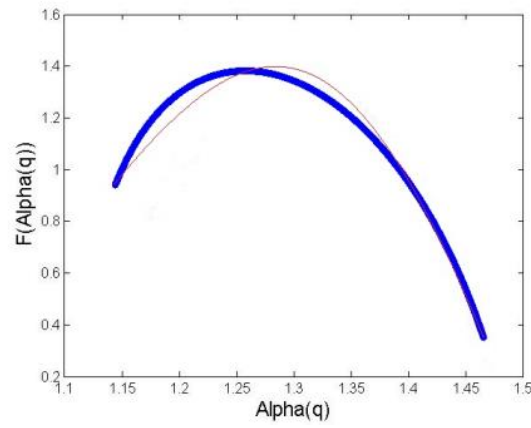

(b)

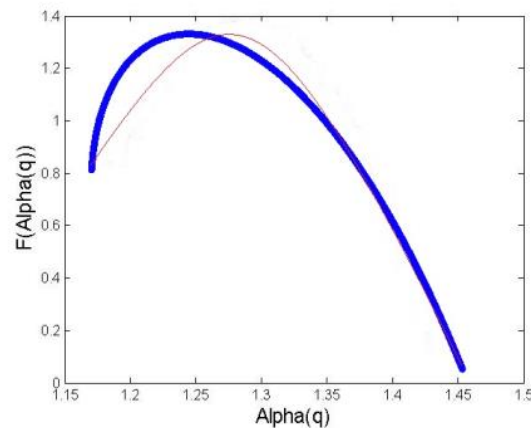

(d)

Figure 14. Four Graphs of Multifractal Spectrum for the Four Fingerprints of Figure 13a-d, respectively

Figure 13 are four different personal fingerprint images, where Figure $13 \mathrm{a}$ is the same fingerprint with Figure 1. The multifractal spectrum curve of these four fingerprint straight segments images are shown in Figure 14. Table 1 shows the multifractal spectrum parameters of these four fingerprint images. Compare the experimental results of Fig. 13a and Fig. 1, we can easily get the four value $\left|\Delta f_{\max }\right|=0.0026,|\Delta W|=0.0330,|\Delta H|=0.0015$ and $|\Delta b|=0.0491$. From Figure 14 and Table 1, we can see that the multifractal spectrum curve and multifractal spectrum parameters of different fingerprint straight segments images are different, while for the same one is similar. 
Table 1. Multifractal Spectrum Parameters for the Four Fingerprints of Figure 13a-d

\begin{tabular}{ccccc}
\hline $\begin{array}{c}\text { Fingerprint } \\
\text { image }\end{array}$ & Maximum $f_{\max }$ & Bandwidth $W$ & Top displacement $H$ & $\begin{array}{c}\text { Asymmetry } \\
\text { parameter } b\end{array}$ \\
\hline Fig. 14(a) & 1.2957 & 0.4203 & 0.0536 & 1.3438 \\
Fig. 14(b) & 1.3933 & 0.3298 & 0.0365 & 1.1327 \\
Fig. 14(c) & 1.3001 & 0.3452 & 0.0491 & 2.1653 \\
Fig. 14(d) & 1.3452 & 0.2968 & 0.0423 & 1.9352 \\
\hline
\end{tabular}

In order to achieve accurate fingerprint identification, we can choose the appropriate threshold parameters by experiments. In this paper, the four thresholds of multifractal spectrum parameters are $\left|\Delta f_{\text {max }}\right| \leq 0.01,|\Delta W| \leq 0.05,|\Delta H| \leq 0.005$ and $|\Delta b| \leq 0.05$. To determine whether different fingerprint straight segments images belong to the same fingerprint is to judge whether they simultaneously satisfy the four thresholds. Only those who can simultaneously satisfy the four thresholds are the same fingerprint.

In order to achieve accurate fingerprint identification, we can choose the appropriate threshold parameters by experiments. In this paper, the four thresholds of multifractal spectrum parameters are $\left|\Delta f_{\max }\right| \leq 0.01,|\Delta W| \leq 0.05,|\Delta H| \leq 0.005$ and $|\Delta b| \leq 0.05$. To determine whether different fingerprint straight segments images belong to the same fingerprint is to judge whether they simultaneously satisfy the four thresholds. Only those who can simultaneously satisfy the four thresholds are the same fingerprint.

\section{Conclusions}

In this paper, we have proposed a novel fingerprint recognition method based on multifractal spectrum theory. The most critical problem in fingerprint recognition is to extract fingerprint features that can distinguish an individual from others. Most of the traditional methods of fingerprint recognition are based on minutiae features, such as ridges and valleys. Although many new fingerprint recognition methods have joined the calculation of orientation field, the accurate extraction of minutiae features is still the fatal flaw for fingerprint recognition.

However, the long digital straight segments instead of the minutiae features of fingerprint in the new multifractal spectrum fingerprint recognition method in this paper. Compared with the minutiae features ridges or valleys, the long digital straight lines are more stable and more reliable. Moreover, as each long digital straight line can be expressed by three parameters and just long straight segments are retained, much less data spaces are needed to store the long straight segments fingerprint features. Finally, this method only needs to use four multifractal spectrum parameters of fingerprint (maximum $f_{\max }$, bandwidth $W$, top displacement $H$ and asymmetry parameter $b$ ) to represent the fingerprint characteristics, which more convenient and more intuitive compared with the traditional minutiae based method.

However, the accuracy of many conditions in the process of fingerprint image acquisition and preprocessing directly affect the accuracy of multifractal spectrum fingerprint recognition. Such as the direction of fingerprint capture, the precise extent of fingerprint core point detection and the accuracy of fingerprint thinning et al. Therefore, the results of this paper should be regarded as preliminary and promising. We have reason to believe that the 
multifractal spectrum method will inject fresh blood to the diversified development of fingerprint identification technology.

\section{Acknowledgements}

This work is supported by the Fundamental Research Funds for the Central Universities (No. 2572014CB30), the Project of "948" program of State Forestry Administration of China (No.2011-4-18), the Project of National Science Foundation of China (No. 31170518), the Project of Natural Science Foundation of Heilongjiang Province of China (No. C201338), and the Project of Science and technology research of department of education of Heilongjiang pr ovince (No.12543019).

\section{References}

[1] S. Jirachaweng, Z. J. Hou, W. Y. Yau and V. Areekul, "Residual orientation modeling for fingerprint enhancement and singular point detection", Pattern Recognition, vol. 44, (2011), pp. 431-442.

[2] M. H. Liu, S. X. Liu and Q. J. Zhao, "Fingerprint orientation field reconstruction by weighted discrete cosine transform", Information Sciences, vol. 268, (2014), pp. 65-77.

[3] X. B. Jiang, X. G. You, Y. Yuan and M. M. Gong, "A method using long digital straight segments for fingerprint recognition", Neurocomputing, vol. 77, (2012), pp. 28-35.

[4] S. G. Hu, Q. M. Cheng, L. Wang and D. Y. Xu, "Modeling land price distribution using multifractal IDW interpolation and fractal filtering method", Landscape and Urban Planning, vol. 110, (2013), pp. 25-35.

[5] L. Yu and D. W. Qi, "Analysis and processing of decayed log CT image based on multifractal theory", Computers and electronics in agriculture, vol. 63, (2008), pp. 147-154.

[6] V. S. Srinivasan and N. N. Murthy, "Detection of singular points in fingerprint images", Pattern Recognition, vol. 25, (1992), pp. 139-153.

[7] J. C. Yang and D. S. Park, "A fingerprint verification algorithm using tessellated invariant moment features", Neurocomputing, vol. 71, (2008), pp. 1939-1946.

[8] R. W. Hall, "Optimally small operator supports for fully parallel thinning algorithms", IEEE Transactions on Pattern Analysis and Machine Intelligence, vol. 15, (1993), pp. 828-833.

[9] N. G. Bourbakis, "A parallel-symmetric thinning algorithm”, Pattern Recognition, vol. 22, (1989), pp. 387396.

[10] G. M Liang and X. J. Cai, "Improvement of OPTA algorithm and its application in fingerprint images thinning", Computer Engineering and Design, vol. 27, (2006), pp. 4607-4609.

[11] J.-P. Reellles and I. Debled-Rennesson, "A linear algorithm for segmentation of digital curves", Pattern Recognition, vol. 9, (1995), pp. 635-662.

[12] H. Zhou, T. Li, Q. J. Xing and Z. Y. Ou, "Identification and segmentation of digital curves", Journal of Dalian University of Technology, vol. 37, (1997), pp. 576-580.

[13] I. D. Rennesson, J. L. Rémy and J. Rouyer-Degli, "Linear segmentation of discrete curves into blurred segments", Discrete Applied Mathematics, vol. 151, (2005), pp. 122-137.

\section{Authors}

Dawei Qi is currently the Dean of the College of Science at Northeast Forestry University, China. He has published more than 110 papers in major international journals and conferences including the IEEE CCDC, WSAT, CAEIA, AISS and JCIT. His research interests are concentrated on wood nondestructive testing, image processing and pattern recognition.

Hongbo Mu is currently an associate professor at Northeast Forestry University, China. $\mathrm{He}$ received the Ph.D. degree in Forest engineering from Northeast Forestry University, China, in 2010. He is the discipline leaders of biological physics in Northeast Forestry University. His main research interests are artificial neural network and wood nondestructive testing. 
International Journal of Hybrid Information Technology Vol.8, No.1 (2015) 